\title{
Intervenções Nutricionais em Síndrome Metabólica: uma Revisão Sistemática
}

\author{
Nutritional Interventions in Metabolic Syndrome: a Systematic Review \\ Leila Sicupira Carneiro de Souza Leão, Milena Miranda de Moraes, Giulia Xavier de Carvalho, \\ Rosalina Jorge Koifman \\ Universidade Federal do Estado do Rio de Janeiro, FIOCRUZ/Escola Nacional de Saúde Pública, Rio de Janeiro, RJ - Brasil
}

\section{Resumo}

Não existe consenso sobre a estratégia nutricional mais adequada para tratar a Síndrome Metabólica (SM), de tal forma que ocorra redução do risco cardiovascular. O presente estudo foi desenhado para avaliar a força de evidência dos benefícios de diferentes intervenções nutricionais na remissão da SM. A busca virtual foi realizada nas bases de dados Medline, Cochrane Library e PubMed, de ensaios clínicos randomizados publicados no período entre 1999 a 2009, em qualquer língua, em indivíduos com 18 anos ou mais e diagnóstico de SM, independente do critério. O operador booleano and foi utilizado na combinação dos MeSH terms "Metabolic Syndrome", "Síndrome x Metabólica" e "Metabolic Syndrome X"; dos entry terms "Dysmetabolic Syndrome X", Metabolic Cardiovascular Syndrome", "Metabolic X Syndrome", "Syndrome X, Metabolic" adicionados dos termos "diet", "intervention and diet", "treatment and diet" e "supplementation". Para cada estudo incluído na revisão foi estimada a Prevalência de SM e o Cálculo da Eficácia após o período de seguimento. Medidas de risco relativo para cada estudo foram descritas pelo Forest Plot. Foram identificados 131 artigos, os quais após critérios de elegibilidade resultaram em 15 estudos. Estes foram separados em quatro grupos: dieta normocalórica associada a exercícios; dieta normocalórica isolada; dieta hipocalórica associada a exercícios; e dieta hipocalórica isolada. Os ensaios com dieta hipocalórica associada à prática de exercícios apresentaram valores mais elevados de eficácia, colaborando para ressaltar os aspectos globais da mudança do estilo de vida no tratamento da SM, onde a alimentação saudável e reduzida em calorias deve ser complementada com a prática de atividade física.

\section{Palavras-chave}

Síndrome metabólica, ensaios clínicos, exercício, dieta com restrição de gorduras, medicina baseada em evidências.

Correspondência: Leila Sicupira Carneiro de Souza Leão • Rua Nicanor Nunes, 102, quadra 17, lote 26, - Piratininga - 24350-460 Niterói, RJ - Brasil

E-mail: leilaleao@gmail.com, rosalina.koifman@hotmail.com Artigo recebido em 05/04/10; revisado recebido em 29/06/10; aceito em 16/08/10.

\section{Introdução}

A Síndrome Metabólica (SM) é definida por um conjunto de ao menos três alterações, seja na pressão arterial ou no metabolismo dos glicídios e lipídios, usualmente associada às doenças cardiovasculares, principal causa de morbimortalidade no Brasil ${ }^{1}$.

Não existe consenso sobre a estratégia nutricional mais adequada para tratar a SM. Embora as propostas atuais estejam relacionadas a mudanças comportamentais como modificação dos hábitos alimentares e prática de atividade física $^{2}$, as recomendações nutricionais foram estabelecidas a partir de estudos realizados com pacientes saudáveis ou que apresentavam as alterações metabólicas isoladas ${ }^{3-8}$. Desta forma, sendo o tratamento eficaz para apenas um componente da síndrome, ao invés da remissão do diagnóstico, representada pela normalização de ao menos três características, a proteção cardiovascular seria consequentemente menor.

Neste contexto, o Projeto Diretrizes da Sociedade Brasileira de Endocrinologia ${ }^{4}$, ressalta a necessidade da realização de pesquisas que contribuam na definição do tipo de intervenção mais eficaz no tratamento de indivíduos com o diagnóstico de SM.

Devido à ausência de revisões sistemáticas sobre o tema, o presente estudo foi desenhado para avaliar a força de evidência dos benefícios de diferentes intervenções nutricionais na remissão da SM.

\section{Métodos}

Foi realizada uma revisão sistemática de estudos com intervenções nutricionais, em indivíduos com SM diagnosticados pela presença de ao menos três alterações conforme preconizado pela Organização Mundial de Saúde em $1999^{9}$. Além desta publicação oficial, outros critérios diagnósticos sugeridos pelos órgãos NCEP-ATP III ${ }^{10}, \mathrm{AHA}^{11}$; $I^{12}$ e $E^{12} I^{13}$, também foram considerados, visto que representavam adaptações ou modificações do critério oficial.

A busca virtual foi realizada nas bases de dados Medline, Cochrane Library e PubMed, de ensaios clínicos publicados no período entre 1999 a 2009, em qualquer língua. A avaliação dos critérios de elegibilidade foi elaborada de forma independente por dois revisores e em caso de divergências, um terceiro pesquisador era consultado.

O operador booleano and foi utilizado na combinação dos MeSH terms "Metabolic Syndrome", "Síndrome x 
Metabólica" e "Metabolic Syndrome X"; dos entry terms "Dysmetabolic Syndrome X", Metabolic Cardiovascular Syndrome", "Metabolic X Syndrome", "Syndrome X, Metabolic" adicionados dos termos "diet", "intervention and diet", "treatment and diet" e "supplementation". O tipo de publicação foi ensaio clínico randomizado para todas as combinações de palavras.

Os resumos dos artigos selecionados foram analisados para verificar se atendiam aos critérios de inclusão que compreendiam: apresentar um delineamento de ensaio clínico controlado com proposta de intervenção nutricional; em população acima de 18 anos de idade; e considerar a remissão da SM como desfecho. Foram excluídos os estudos com intervenções destinadas a tratar somente as alterações isoladas da SM.

$\mathrm{Na}$ medida do possível, aderimos ao PRISMA $^{14}$ para relatar a presente revisão sistemática. Após leitura dos artigos completos, os resultados foram sintetizados segundo o critério diagnóstico da SM, a intervenção nutricional, o grupo controle, o período da intervenção e a prevalência de SM, desfecho principal desta revisão.

Para facilitar a comparação entre os estudos, foi calculada a eficácia das intervenções por meio dos valores de prevalência de SM após o período de seguimento, aplicados na fórmula proposta por Szklo e Javier Nieto ${ }^{15}$ : (prevalência no grupo controle prevalência grupo intervenção/prevalência grupo controle) x 100 .

Medidas de risco relativo para cada estudo foram descritas pelo Forest Plot elaborado no software estatístico Review
Manager versão 5. Contudo, a meta-análise estatística não foi justificada por causa da acentuada heterogeneidade dos estudos incluídos.

\section{Resultados}

Na busca eletrônica, foram obtidas 131 publicações, das quais após leitura dos resumos e exclusão por incluir medicamentos, 59 artigos foram selecionados para análise. Após exclusão de 44 artigos que não atenderam aos critérios de inclusão, 15 estudos foram incluídos na revisão (Figura 1).

Todos os estudos selecionados eram ensaios clínicos randomizados e foram publicados na língua inglesa. Quanto ao local de realização, Itália ${ }^{16-18}$ contribuiu com três estudos, Estados Unidos da América ${ }^{19-23}$ com cinco, Finlândia ${ }^{24,25}$ com dois e Japão ${ }^{26}$, Alemanha ${ }^{27}$, Irã ${ }^{28}$, Espanha ${ }^{29}$ e Noruega ${ }^{30}$ com um cada.

As intervenções apresentaram períodos entre 6 semanas e 31 meses, e envolveram 2089 participantes entre 24 e 75 anos, com Índice de Massa Corporal entre 26 e $43 \mathrm{~kg} / \mathrm{m}^{2}$. Dos quinze estudos, somente três citaram período de run in ${ }^{16,25,28}$, e dois com intervenção parcialmente cega ${ }^{17,20}$.

Quanto à população, quatro ensaios pesquisaram pacientes ambulatoriais provenientes de apenas 1 hospital ${ }^{18,22}$, dois foram multicêntricos ${ }^{24,29}$; dois com população representativa de comunidades ${ }^{16,30}$; três com pacientes captados por anúncios de jornais ${ }^{19,25,26}$; um com funcionários de universidades ${ }^{20}$ e três não informaram a população estudada ${ }^{21,23,27}$.

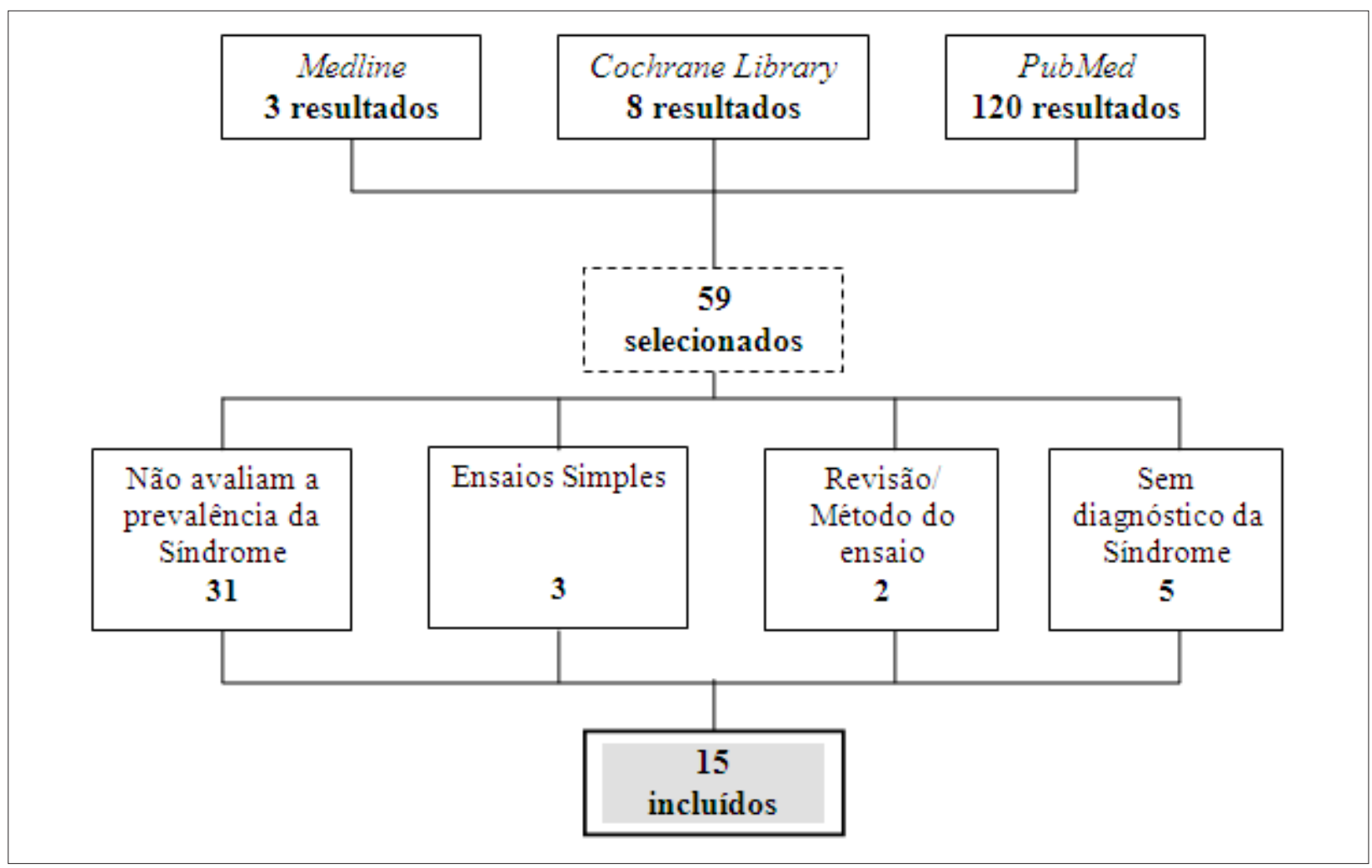

Fig. 1 - Esquema da busca eletrônica e inclusão dos artigos do estudo. 


\section{Artigo de Revisão}

A prevalência de SM foi o desfecho principal em seis estudos ${ }^{16,24-26,29,30}$, e secundária onde a prevalência dos componentes da $\mathrm{SM}^{18-22,27,28}$, a redução ponderal ${ }^{23}$ e $\mathrm{o}$ comportamento dos marcadores inflamatórios e de função endotelial ${ }^{17}$ foram os desfechos principais.

Os ensaios foram agrupados segundo o tipo de intervenção, a saber: dieta normocalórica combinada a exercício físico ${ }^{16,19}$; dieta normocalórica ${ }^{20,29}$; dieta hipocalórica combinada a exercício físico ${ }^{24,25}$; e dieta hipocalórica ${ }^{22,27}$ (Tabela 1).

\section{Dieta normocalórica combinada à exercício físico}

No estudo de Stewart e cols. ${ }^{19}$, o grupo intervenção foi orientado a praticar exercícios aeróbicos supervisionados três vezes por semana, além da dieta individualizada. Os controles receberam apenas orientações gerais sobre alimentação saudável. De forma semelhante Bo e cols. ${ }^{16}$ orientaram seus controles com informações gerais, enquanto o grupo intervenção recebeu orientação nutricional individualizada e recomendação de exercícios.

\section{Dieta normocalórica}

No estudo de Mutungi e cols. ${ }^{20}$, a intervenção foi caracterizada por dieta sem restrição energética, com baixo teor de carboidratos e acrescida de três ovos líquidos (Vistar $\left.{ }^{\circledR}\right)$ por dia. O grupo controle consumiu somente uma dieta de manutenção. Vale destacar que, embora a proposta dietética fosse normocalórica, ambos os grupos reduziram o conteúdo energético da dieta até o final do seguimento.

No ensaio de Salas-Salvadó e cols. ${ }^{29}$, os dois grupos de intervenção consumiram uma dieta mediterrânea suplementada com azeite de oliva extra-virgem $(1,25 \mathrm{~L}$ por semana), ou com oleaginosas (15g nozes/dia; $7,5 \mathrm{~g}$ avelãs/dia; $7,5 \mathrm{~g}$ amêndoas/dia). O grupo controle foi orientado somente com dieta normocalórica.

\section{Tabela 1 - Resumo dos estudos incluídos na revisão sistemática com respectivos cálculos de eficácia}

\begin{tabular}{|c|c|c|c|c|c|}
\hline $\begin{array}{l}\text { Autor } \\
\text { Ano }\end{array}$ & $\begin{array}{c}\text { Critério } \\
\text { Diagnóstico da SM }\end{array}$ & Grupo Intervenção* & Grupo Controle* & $\begin{array}{l}\text { Duração } \\
\text { (meses) }\end{array}$ & $\begin{array}{c}\text { Eficácia do } \\
\text { estudo }\end{array}$ \\
\hline $\begin{array}{l}\text { Stewart e cols. }{ }^{19} \\
2005\end{array}$ & $\begin{array}{l}\text { NCEP/ATPIII } \\
2001\end{array}$ & $\begin{array}{l}\text { Normocalórica + exercícios } \\
\qquad(\mathrm{n}=22)\end{array}$ & $\begin{array}{l}\text { Orientações gerais } \\
\qquad(\mathrm{n}=22)\end{array}$ & 6 & $27,8 \%$ \\
\hline $\begin{array}{l}\text { Bo e cols. }{ }^{16}, \\
2007\end{array}$ & $\begin{array}{l}\text { NCEP/ATPIII } \\
2001\end{array}$ & $\begin{array}{l}\text { Normocalórica + exercícios } \\
\qquad(n=119)\end{array}$ & $\begin{array}{l}\text { Orientações gerais } \\
\qquad(n=120)\end{array}$ & 12 & $46,9 \%$ \\
\hline $\begin{array}{l}\text { Mutungi e cols. }{ }^{20} \text {, } \\
2008\end{array}$ & $\begin{array}{l}\text { NCEP/ATPIII } \\
2001\end{array}$ & $\begin{array}{l}\text { Normocalórica }+3 \text { ovos } \\
\qquad(n=11)\end{array}$ & $\begin{array}{l}\text { Normocalórica } \\
\qquad(n=7)\end{array}$ & 3 & $100 \%$ \\
\hline $\begin{array}{l}\text { Salas-Salvadó e cols. }{ }^{29} \text {, } \\
2008\end{array}$ & $\begin{array}{l}\text { NCEP/ATPIII } \\
2001\end{array}$ & $\begin{array}{c}\text { Normocalórica + azeite } \\
(n=250) \\
\text { Normocalórica + oleaginosas } \\
(n=250)\end{array}$ & $\begin{array}{l}\text { Normocalórica } \\
\qquad(n=250)\end{array}$ & 12 & $\begin{array}{l}5,3 \% \\
13,8 \%\end{array}$ \\
\hline $\begin{array}{l}\text { Kukkonen-Harjula e } \\
\text { cols. }^{25} \\
2005\end{array}$ & $\begin{array}{l}\text { NCEP/ATPIII } \\
2001 \text { modificado }\end{array}$ & $\begin{array}{l}\text { Hipocalórica + exercícios } \\
\qquad(n=10)\end{array}$ & $\begin{array}{l}\text { Hipocalórica } \\
\qquad(n=13)\end{array}$ & 31 & $25,7 \%$ \\
\hline $\begin{array}{l}\text { Villareal e cols. }{ }^{21}, \\
2006\end{array}$ & $\begin{array}{l}\text { NCEP/ATPIII } \\
2001\end{array}$ & $\begin{array}{l}\text { Hipocalórica + exercícios } \\
\qquad(n=9)\end{array}$ & $\begin{array}{l}\text { Orientações gerais } \\
\qquad(n=15)\end{array}$ & 6,5 & $66,7 \%$ \\
\hline $\begin{array}{l}\text { Okura e cols. }{ }^{26} \text {, } \\
2007\end{array}$ & $\begin{array}{l}\text { COMITÊ JAPONÊS } \\
2005\end{array}$ & $\begin{array}{l}\text { Hipocalórica + exercícios } \\
\qquad(n=38)\end{array}$ & $\begin{array}{l}\text { Hipocalórica } \\
\qquad(n=21)\end{array}$ & 3,5 & $81,5 \%$ \\
\hline $\begin{array}{l}\text { Anderssen e cols. }{ }^{30} \text {, } \\
2007 \text {, }\end{array}$ & IDF, 2006 & $\begin{array}{l}\text { Hipocalórica + exercícios } \\
\qquad(n=43)\end{array}$ & $\begin{array}{l}\text { Hipocalórica } \\
\qquad(\mathrm{n}=34)\end{array}$ & 12 & $49,6 \%$ \\
\hline $\begin{array}{l}\text { llanne-Parikka e cols. }{ }^{24} \text {, } \\
2008\end{array}$ & AHA, 2005 & $\begin{array}{l}\text { Hipocalórica + exercícios } \\
\qquad(n=196)\end{array}$ & $\begin{array}{l}\text { Orientações gerais } \\
\qquad(n=190)\end{array}$ & 12 & $14,3 \%$ \\
\hline $\begin{array}{l}\text { Seshadri e cols. }{ }^{22} \text {, } \\
2004\end{array}$ & $\begin{array}{l}\text { NCEP/ATPIII } \\
2001\end{array}$ & $\begin{array}{l}\text { Hipocalórica hipoglicídica } \\
\qquad(n=19)\end{array}$ & $\begin{array}{l}\text { Hipocalórica } \\
\quad(n=17)\end{array}$ & 6 & $16,4 \%$ \\
\hline $\begin{array}{l}\text { Esposito e cols. }{ }^{17} \text {, } \\
2004\end{array}$ & $\begin{array}{l}\text { NCEP/ATPIII } \\
2001\end{array}$ & $\begin{array}{l}\text { Hipocalórica Mediterrânea } \\
\qquad(\mathrm{n}=90)\end{array}$ & $\begin{array}{l}\text { Orientações gerais } \\
\qquad(n=90)\end{array}$ & 24 & $48,8 \%$ \\
\hline $\begin{array}{l}\text { Azadbakht e cols. }{ }^{28} \text {, } \\
2005\end{array}$ & $\begin{array}{l}\text { NCEP/ATPIII } \\
2001\end{array}$ & $\begin{array}{l}\text { Hipocalórica DASH } \\
\qquad(n=38)\end{array}$ & $\begin{array}{l}\text { Hipocalórica } \\
\qquad(\mathrm{n}=38)\end{array}$ & 6 & $19,8 \%$ \\
\hline $\begin{array}{l}\text { Muzio e cols. }{ }^{18} \\
2007\end{array}$ & $\begin{array}{l}\text { NCEP/ATPIII } \\
2001\end{array}$ & $\begin{array}{l}\text { Hipocalórica hipoglicídica } \\
\qquad(n=50)\end{array}$ & $\begin{array}{l}\text { Hipocalórica hiperglicídica } \\
\qquad(\mathrm{n}=50)\end{array}$ & 5 & $23,3 \%$ \\
\hline $\begin{array}{l}\text { Katcher e cols. }{ }^{23} \text {, } \\
2008\end{array}$ & $\begin{array}{l}\text { NCEP/ATPIII } \\
2001\end{array}$ & $\begin{array}{l}\text { Hipocalórica com cereais integrais } \\
\qquad(n=24)\end{array}$ & $\begin{array}{l}\text { Hipocalórica } \\
\qquad(\mathrm{n}=23)\end{array}$ & 3 & $0 \%$ \\
\hline $\begin{array}{l}\text { König e cols. }{ }^{27} \text {, } \\
2008\end{array}$ & $\begin{array}{l}\text { NCEP/ATPIII } \\
2001\end{array}$ & $\begin{array}{l}\text { Hipocalórica + substituto de refeição } \\
\text { à base de soja } \\
(n=22)\end{array}$ & $\begin{array}{l}\text { Hipocalórica } \\
\qquad(n=8)\end{array}$ & 1,5 & $11,9 \%$ \\
\hline
\end{tabular}

NCEP/ATPIII: National Cholesterol Education Program Adult Treatment Panel; IDF - International Diabetes Federation; AHA - American Heart Association; DASH - Dietary Approaches to Stop Hypertension ; *indivíduos com diagnostico de Síndrome Metabólica. 
Dieta hipocalórica combinada a exercício físico

O ensaio de Kukkonen-Harjula e cols. ${ }^{25}$ submeteu os participantes a duas fases: uma redução ponderal e outra de manutenção de peso com a intervenção de interesse, a dieta acrescida de caminhada, e um grupo controle somente com a dieta. No estudo de Villareal e cols. ${ }^{21}$, a intervenção consistia de dieta hipocalórica associada a exercícios, enquanto ao grupo controle consumia apenas a dieta hipocalórica. Okura e cols. ${ }^{26}$ ofertaram aos controles dieta hipocalórica com um suplemento alimentar (MicroDiet ${ }^{\circledR}$ ). O grupo intervenção consumiu a mesma dieta associada à prática de exercícios.

No estudo de Anderssen e cols. ${ }^{30}$ o grupo do exercício combinado à dieta foi supervisionado para realizar atividade aeróbica três vezes por semana e consumo calórico com restrição energética. Os controles consumiram apenas a dieta hipocalórica individualizada.

No ensaio de llanne-Parikka e cols. ${ }^{24}$ a intervenção consistiu de orientações para aumentar o nível de atividade física, acréscimo de $15 \mathrm{~g}$ de fibras para cada $1000 \mathrm{kcal}$ e adoção de dieta com baixo teor de gordura e colesterol. O grupo controle foi abordado apenas com orientações gerais.

\section{Dieta hipocalórica}

O ensaio de Seshadri e cols. ${ }^{22}$ ofertou dieta hipocalórica balanceada para o grupo controle enquanto o intervenção foi orientado a consumir dieta hipocalórica pobre em carboidratos. Esposito e cols. ${ }^{17}$ orientaram o grupo intervenção com dieta mediterrânea, enquanto o controle orientações gerais sobre alimentação saudável.

No estudo de Azadbakht e cols. ${ }^{28}$, a intervenção consistia na dieta DASH (Dietary Approaches to Stop Hypertension), com déficit de $500 \mathrm{kcal} / \mathrm{dia}$, maior ingestão de frutas, vegetais, laticínios desnatados e menor teor de sódio.

Muzio e cols. ${ }^{18}$ realizaram intervenção com $48 \%$ de carboidratos e o controle com $65 \%$, ambos com déficit de 500 kcal/dia e sessões mensais em grupo para orientação nutricional e Katcher e cols. ${ }^{23}$ compararam um grupo de dieta hipocalórica à base de cereais integrais com outro que consumiu cereais refinados.

O ensaio de König e cols. ${ }^{27}$ orientou o grupo controle a consumir dieta hipocalórica e o grupo intervenção a substituir duas refeições diárias por um iogurte de soja com mel $\left(\right.$ Almased $\left.{ }^{\circledR}\right)$

Considerando todos os estudos incluídos na revisão, bem como os diferentes agrupamentos segundo tipo de intervenção e correspondente cálculo da eficácia, observou-se que a dieta hipocalórica associada aos exercícios físicos apresentou melhor desempenho em relação aos outros grupos, seguida pela normocalórica com exercícios, hipocalórica isolada e normocalórica isolada.

Contudo, independente do tipo de intervenção nutricional, as medidas de efeito expressas no Forest Plot descrevem efeito protetor no risco de desenvolver SM para todas as propostas de tratamento nutricional analisadas (Figura 2).

\section{Discussão}

Até quanto se conhece esta é a única revisão sistemática que avaliou a força de evidência de intervenções nutricionais na remissão do diagnóstico de SM. Após uma extensa estratégia de busca, a inclusão de apenas 15 estudos expressou a escassez da literatura quando se trata de avaliar o diagnóstico da SM como desfecho categórico principal ou secundário.

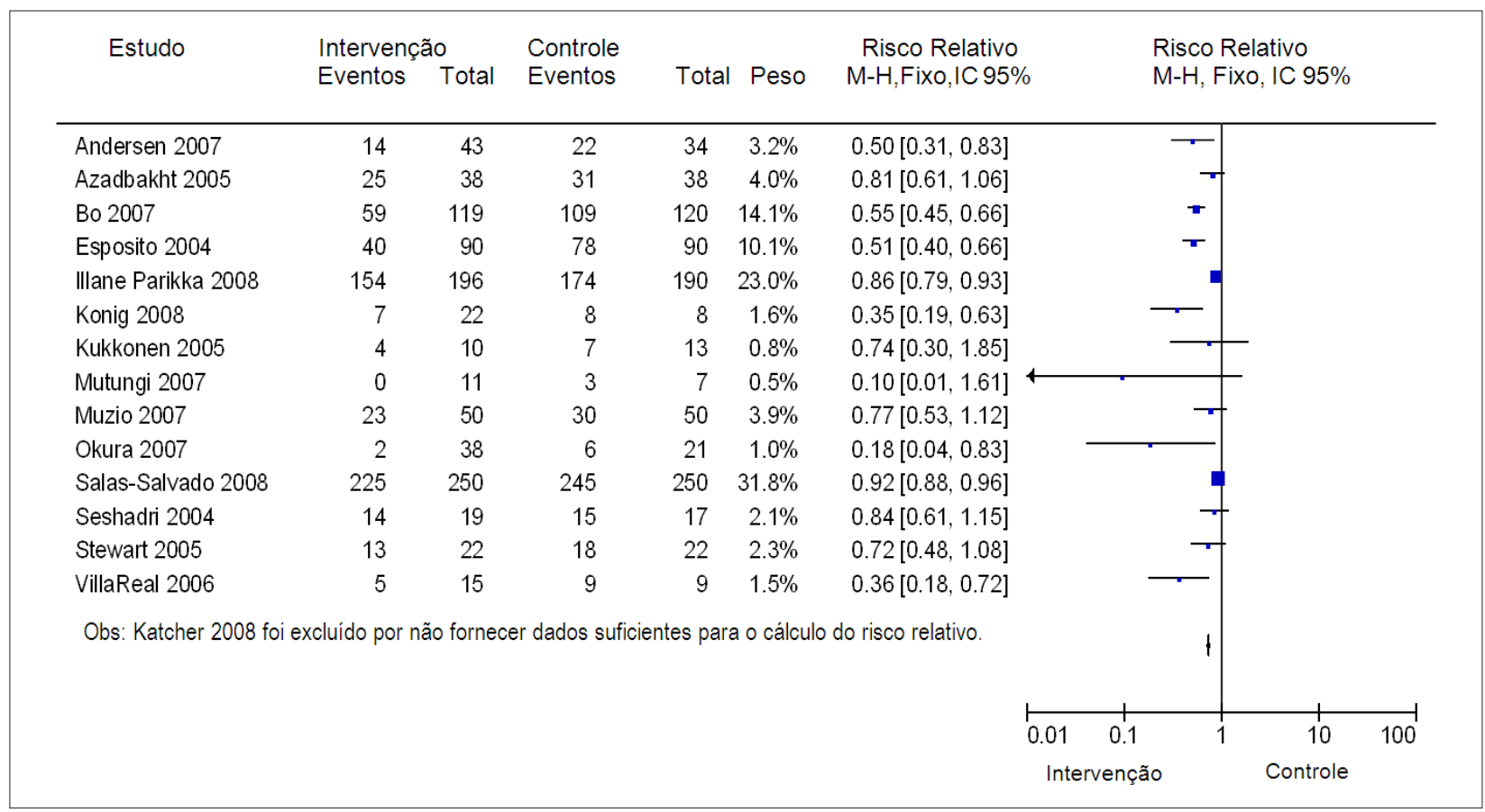

Fig 2 - Forest Plot dos estudos incluídos na Revisão Sistemática e suas respectivas medidas de associação. 


\section{Artigo de Revisão}

Além da limitação quantitativa, a baixa qualidade e heterogeneidade dos estudos também foi notada. Nenhum autor conduziu o ensaio segundo o guia CONSORT (http:// www.consort-statement.org/), houve elevada variabilidade quanto ao tempo de intervenção, alguns estudos desenharam grupos controle baseados apenas em orientações gerais, enquanto outros dieta calculada, e a maioria, comparou grupos com amostras pequenas ( $<30$ participantes).

Estas características, associadas ao pobre detalhamento da metodologia na maioria dos artigos impediram esclarecimento sobre o cálculo amostral, bem como informações específicas do desenho da intervenção proposta como cegamento e período de run in, impedindo uma metaanálise dos resultados apresentados.

Contudo, a eficácia das propostas hipocalóricas associadas à atividade física foi semelhante às intervenções realizadas em indivíduos com as alterações isoladas ${ }^{31-34}$, e reforçam a sugestão de Reaven ${ }^{35}$, que propôs uma dieta reduzida em calorias associada à atividade física para o tratamento de pacientes com SM.

Pode-se considerar que a redução calórica da dieta é a principal estratégia para o tratamento do excesso de peso ${ }^{36}$, porque os aportes calóricos reduzidos promovem a diminuição da massa adiposa através de balanço energético negativo, condição na qual o gasto energético supera o consumo de energia. Quando a redução calórica é adicionada da prática de atividades físicas, o efeito da redução ponderal é potencializado, especialmente em indivíduos com SM, onde a manutenção da massa magra aumentaria a sensibilidade à insulina ${ }^{37}$.

A importância da atividade física agregada a um planejamento alimentar ainda que normocalórico foi demonstrada nos estudos de Stewart e cols. ${ }^{19}$ e Bo e cols. ${ }^{16}$. Mesmo com resultados de eficácia inferiores às intervenções com dieta hipocalórica e exercícios, as características semelhantes de idade e IMC em ambos os grupos e a manutenção do consumo energético pós intervenção reforçam a influência da atividade física na redução ponderal. Ao mesmo tempo, a reduzida eficácia da dieta normocalórica não associada à atividade física de Salas-Salvadó e cols. ${ }^{29}$, confirmou a necessidade de um balanço energético negativo no tratamento da SM, seja por redução calórica ou por aumento do gasto energético.

Neste sentido, pode-se compreender as sugestões do Projeto Diretrizes ${ }^{4}$ que valoriza a necessidade de redução do conteúdo energético das propostas de tratamento para SM, em detrimento às mudanças na composição em macronutrientes das mesmas. Para isso, recomendamos que ensaios clínicos controlados randomizados e de alta qualidade sejam conduzidos para detectar os efeitos das exposições dietéticas com segurança e direcionar abordagens mais objetivas na medição do consumo dietético e desfechos de relevância na saúde pública.

A presente revisão sistemática apresentou limitações como uma provável não identificação de todas as publicações, apesar da tentativa de minimizar esta possibilidade por meio da aplicação de termos amplos, da repetição da busca nas bases de dados e nas referências dos artigos selecionados. Como pontos fortes destacamos a abordagem sistemática, a ampla busca em relevantes bases de dados, a inclusão de publicações em qualquer língua, e o rigor metodológico.

Embora o conjunto de intervenções com dieta hipocalórica associada à atividade física tenha apresentado resultados que se destacaram em relação aos outros tipos de propostas nutricionais, a análise conjunta dos estudos forneceu evidência dos benefícios do consumo de dietas individualizadas, pobres em gorduras saturadas, ricas em fibras, óleos monoinsaturados, vitaminas e minerais na remissão da Síndrome Metabólica.

\section{Conclusão}

Mesmo levando em consideração fatores que limitaram esta revisão sistemática, pode-se concluir que a literatura científica sustenta os efeitos benéficos da dieta hipocalórica associada à prática de exercícios físicos, reforçando a importância da mudança do estilo de vida no manejo da SM.

\section{Potencial Conflito de Interesses}

Declaro não haver conflito de interesses pertinentes.

\section{Fontes de Financiamento}

O presente estudo foi parcialmente financiado pela UNIRIO e FIOCRUZ.

\section{Vinculação Acadêmica}

Este artigo é parte de tese de Doutorado de Leila Sicupira Carneiro de Souza Leão pela Escola Nacional de Saúde Pública - FIOCRUZ.

\section{Referências}

1. Ministério da Saúde. Secretaria de Vigilância em Saúde. Uma análise da situação de saúde. Perfil de Mortalidade do Brasileiro. Brasília, 6 de novembro de 2008. [Citado em 2008 nov 8]. Disponível em: http://www.saude.gov.br.

2. Deen D. Metabolic syndrome: time for action. Am Fam Physician. 2004; 69 (12): 2875-82.

3. Carvalho MHC, Brandão AP, Brandão AA, Nogueira AR, Suplicy $H$, Guimarães JI, et al. / Sociedade Brasileira de Hipertensão / Sociedade
Brasileira de Cardiologia / Sociedade Brasileira de Endocrinologia e Metabologia / Sociedade Brasileira de Diabetes. I Diretriz brasileira de diagnóstico e tratamento da síndrome metabólica. Arq Bras Cardiol. 2005; 84 (supl 1): 3-28.

4. Sociedade Brasileira de Endocrinologia e Metabologia. Projeto diretrizes: Síndrome Metabólica: tratamento não-farmacológico para redução do risco cardiovascular. 2006. [citado em 2008 ago 19]. Disponível em: http://www. projetodiretrizes.org.br/5_volume/38-SindrMeta.pdf. 
5. Hooper L, Bartlett C, Davey SG, Ebrahim S. Advice to reduce dietary salt for prevention of cardiovascular disease. Cochrane Databse Syst Rev. 2004; (1): CD003656.

6. Hooper L, Summerbell CD, Higgins JP, Thompson RL, Clements G, Capps N, et al. Reduced or modified dietary fat for preventing cardiovascular disease. Cochrane Database Syst Rev. 2001; (3): CD002137.

7. Vollmer WM, Sacks FM, Ard J, Appel LJ, Bray GA, Simon-Morton DG, et al. Effects of diet and sodium intake on blood pressure: subgroup analysis of the DASH-sodium trial. Ann Intern Med. 2001; 135 (12): 1019-28.

8. Trichopoulou A, Costacou T, Bamia C, Trichopoulos D. Adherence to a Mediterranean diet and survival in a Greek population. N Engl J Med. 2003; 348 (26): 2599-608.

9. World Health Organization. Definition, diagnosis and classification of diabetes mellitus and its complications: report of a WHO consultation. Washington; 1999.

10. Executive Summary of the Third Report of the National Cholesterol Education Program (NECP). Expert Panel on Detection Evaluation, and Treatment of High Blood Cholesterol in Adults (Adult Treatment Panel III). JAMA. 2001; 285 (19): 2486-97.

11. Grundy SM, Brewer HB, Cleeman II, Smith SC, Lenfant C. Definition of metabolic syndrome: report of the National Heart, Lung, and Blood Institute/ American Heart Association conference on scientific issues related to definition. Arterioscler Thromb Vasc Biol. 2004; 24 (2): e13-8.

12. International Diabetes Federation. [homepage na Internet]. The IDF consensus worldwide definition of the Metabolic Syndrome. [citado 2008 out 10]. Disponível em http://www.idf.org.

13. Balkau B, Charles MA. Comment on the provisional report from the WHO Consultation. European Group of the Study of Insulin Resistance (EGIR). Diabet Med.1999; 16 (5): 442-3.

14. Moher D, Liberati A, Tetzlaff J, Altman DG. Preferred reporting items for systematic reviews and meta-analyses: the PRISMA statement. J Clin Epidemiol. 2009; 62 (10): 1006-12.

15. Szklo M, Javier Nieto, F. Epidemiology beyond the basics. Maryland: Aspen Publication; 2000 .

16. Bo S, Ciccone G, Baldi C, Benini L, Dusio F, Forastiere G, et al. Effectivenes of a lifestyle intervention on metabolic syndrome: a randomized controlled trial. J Gen Intern Med. 2007; 22 (12): 1695-703.

17. Esposito K, Marfella R, Ciotola M, Di Palo C, Giugliano F, Giugliano G, et al. Effect of a Mediterranean-Style diet on endothelial dysfunction and markers of vascular inflammation in the metabolic syndrome. JAMA. 2004; 292 (12): $1440-6$.

18. Muzio F, Mondazzi L, Harris WS, Sommariva D, Branchi A. Effects of moderate variations in the macronutrient content of the diet on cardiovascular disease risk factors in obese patients with the metabolic syndrome. Am J Clin Nutr. 2007; 86 (4): 946-51.

19. Stewart KJ, Bacher AC, Turner K, Lim JG, Hees PS, Shapiro EP, et al. Exercise and risk factors associated with metabolic syndrome in older adults. Am J Prev Med. 2005; 28 (1): 9-18.

20. Mutungi G, Ratliff, Puglisi M, Torres-Gonzalez M, Vaishnav U, Leite JO, et al. Dietary cholesterol from eggs increases plasma HDL cholesterol in overweight men consuming a carbohydrate-restricted diet. J Nutr. 2008; 138 (2): 272-6.

21. Villareal DT, Miller BV, Banks M, Fontana L, Sinacore DR, Klein S. Effect of lifestyle intervention on metabolic coronary heart disease risk factors in obese older adults. Am J Clin Nutr. 2006; 84 (6): 1317-23.
22. Seshadri P, Iqbal N, Stern L, Williams M, Chicano KL, Daily DA, et al. A randomized study comparing the effects of a low-carbohydrate diet and a conventional diet on lipoprotein subfractions and C-reactive protein levels in patients with severe obesity. Am J Med. 2004; 117 (6): 398-405.

23. Katcher HI, Legro RS, Kunselman AR, Gillies PI, Demers LM, Bagshaw DM, et al. The effects of a whole grain-enriched hypocaloric diet on cardiovascular disease risk factors in men and women with metabolic syndrome. Am J Clin Nutr. 2008; 87 (1): 79-90.

24. Ilanne-Parikka P, Eriksson JG, Lindström J, Peltonen M, Aunola S, Hämäläinen $\mathrm{H}$, et al. Effect of lifestyle intervention on the occurrence of metabolic syndrome and its components in the Finnish Diabetes Prevention Study. Diabetes Care. 2008; 31 (4): 805-7.

25. Kukkonen-Harjula KT, Borg PT, Nenonen AM, Fogelholm MG. Effects of a weight maintenance program with or without exercise on the metabolic syndrome: a randomized trial in obese men. Prev Med. 2005; 41 (3-4): 784 90.

26. Okura T, Nakata Y, Ohkawara K, Numao S, Katayama Y, Matsuo T, etal. Effects of aerobic exercise on metabolic syndrome improvement in response to weight reduction. Obesity. 2007; 15 (10): 2478-84.

27. König D, Deibert P, Frey I, Landmann U, Berg A. Effect of meal replacement on metabolic risk factors in overweight and obese subjects. Ann Nutr Metab. 2008; 52 (1): 74-8.

28. Azadbakht L, Mirmiran P, Esmaillzadeh A, Azizi T, Azizi F. Beneficial effects of a dietary approaches to stop hypertension eating plan on features of the metabolic syndrome. Diabetes Care. 2008; 28 (12): 2823-31.

29. Salas-Salvadó J, Fernandez-Ballart J, Ros E, Martinez-Gonzalez MA, Fitó M Estruch R, et al. Effect of a Mediterranean diet supplemented with nuts on metabolic syndrome status: one-year results of the PREDIMED Randomized Trial. Arch Intern Med. 2008; 168 (22): 2449-58.

30. Anderssen SA, Carroll S, Urdal P, Holme I. Combined diet and exercise intervention reverses the metabolic syndrome in middle-aged males: results from the Oslo Diet and Exercise Study. Scand J Med Sci Sports. 2007; 17 (6): $687-95$.

31. Jacobs DR, Sluik D, Rockling-Andersen MH, Anderssen SA, Drevon CA Association of 1-y changes in diet pattern with cardiovascular disease risk factors and adipokines: results from the 1-y randomized Oslo Diet and Exercise Study. Am J Clin Nutr. 2009; 89 (2): 509-17.

32. Kuller LH, Kinzel LS, Pettee KK, Kriska AM, Simkin-Silverman LR, Conroy MB et al. Lifestyle intervention and coronary heart disease risk factor changes ove 18 months in postmenopausal women: the women on the move through activity and nutrition (Woman Study) clinical trial. J Womens Health. 2006; 15 (8): 962-74.

33. Meckling KA, Sherfey R. A randomized trial of a hypocaloric high-protein diet, with and without exercise, on weight loss, fitness, and markers of the metabolic syndrome in overweight and obese women. Appl Physiol Nutr Metab. 2007; 32 (4): 743-52

34. Oh EG, Hyun SS, Kim SH, Bang SY, Chu SH, Jeon JT, et al. A randomized controlled trial of therapeutic lifestyle modification in rural women with metabolic syndrome: a pilot study. Metabolism. 2008; 57 (2): 255-61.

35. Reaven G. Banting lecture 1988: role of insulin resistance in human disease. Diabetes. 1988; 37 (12): 1595-607.

36. Francischi RPP, Pereira LO, Freitas CS. Obesidade: atualização sobre etiologia morbidade e tratamento. Rev Nutr. 2000; 13: 17-28.

37. Lyra R, Oliveira M, Lins D, Cavalcanti N. Prevenção do diabetes mellitus tipo 2. Arq Bras Endocrinol Metab. 2006; 50 (2): 239-49. 